assures us will be printed in exceptionally quick time and will be largely complete, will be a valuable reference document, giving hard data on the projects as well as construction and operational programmes.

One of these days however, the physics community will need to discuss priorities not only in, but between fields as the total budget envelope becomes more restricting and as more governments begin to think of science budgets as entities and look to the scientific establishment to define its priorities. If it refuses to do so then it must not complain when, as has happened in the USA, civil servants and politicians do it instead. The necessity for such discussions was underlined by the one lecture that touched on the subject given by $\mathrm{J}$. Irvine of the Sussex University Science Policy Research Unit. Despite the care with which he developed his theme, there was a strong gut reaction from several commentators asserting that Irvine did not understand what pure research was about and that he had no business to touch the sacred cow. This ignored the thrust of his talk which was that "the big science community could do much to counter this threat (of cuts in budgets) by becoming more concerned with wider research policy issues when proposing new initiatives". Unfortunately science is all too prone to be unscientific when discussing its own funding. How many really accepted the figure that from 1980-87, spending on university and related research in six leading OECD nations rose in real terms by over $20 \%$ on average.

The trigger was probably the quotation from Frank Press who maintained before the US National Academy of Sciences that they had an unprecedented number of superb research proposals on the table and because of the need for choice, larger projects like the SSC had a second-order claim on restricted funds, a view that seems to be shared by the Joint Congressional Committee's research unit. Even in the corridors in Zagreb, the frequent comment had been that if Texas is prepared to sink a billion dollars in the SSC and central government has apportioned $\$ 100 \mathrm{M}$ for this year, let them get on with it. Irvine's point was that such specious conclusions distorted the whole field, especially in the light of under-funding of SLAC and the write-off of $\$ 200 \mathrm{M}$ on Isabelle. The USA, it is recognised, has a problem because of intense inter-laboratory competition that paralyses debates on scientific priorities. Sad that Europe's only vocal reaction should be confined

to a strident cry of leave high energy physics alone. This may not be the best thing - for high energy physics. If physicists cannot argue a case amongst themselves, they become wide open to outside attacks based on the principle of divide and rule.

Next time, perhaps we ought to try a little evaluation and not just have a shop

\title{
Applied Physics in Nice
}

To coincide with the 9th General Conference of the Condensed Matter Division, the EPS Advisory Committee on Applied Physics and Physics in Industry (ACAPPI) met in March and also had discussions with delegates of the Associate Members. All participants had been invited to attend the closing session of the conference and hear the summary of Professor J. Friedel.

As introduction, Friedel asserted that condensed matter physics was clearly doing fine. Far from running out of interest it was burgeoning in many directions and its applications were multiplying. Amongst the examples he chose to illustrate this contention, were the multilayer semiconductors that were giving rise to new devices, the high $T_{c}$ superconductors finding their initial use in microtechnics, and also the adoption of "academic" reasoning to "practical" problems - going up the scale from microscopic thinking, on percolation for example, to macroscopic situations and using medium size techniques in such areas as plasticity and fracture. Hydrodynamics and turbulence were also benefiting and even such global aspects as volcano behaviour and climate. The overlap between fields of physics was very evident with parallel computing being used to analyse neural networks, the structure of clusters echoing nuclear physics and so on. There was also a great development of concepts - localisation by disorder applied to phonon frustration and charge density waves; scaling up of defects from short range interactions, through the creation of blocks that then can be treated as entities and on up to the behaviour of massive bodies.

The border line between pure and applied physics, if such a one exists, was evidently very vague, a fact much debated in ACAPPI. One should be aware, however, that by nature the applied physicist felt less urge to appear in print or on the rostrum than the colleague in pure physics. He (or she) will often present research results only in a company or institute report.

This cultural difference should be taken into account by "pure" physicists complaining about the laziness of their colleagues in applied work, and by industrial physicists grumbling that, judging from its public manifestations, the EPS is clearly dominated by the academics. For the Society it means that to involve an applied physicist in its activities will often require an extra effort. window of goodies with the price tags discretely hidden from view.

That said, one must congratulate the local organising committee on the practical arrangements, and thank the President of the Republic of Croatia for our one bit of relaxation - the generous reception in his quite beautiful palace in the old town quarter of Zagreb.

\section{Photovoltaics}

One of those extra efforts was the meeting in Nice at which Professor M. Rodot gave a talk (followed by many questions) on Photovoltaics, a field which has moved from the laboratory into becoming a sizable industry. In 1987 (the latest for which figures were available) some $30 \mathrm{MW}$ of capacity was in operation in the USA representing an investment of $0.3 \mathrm{G} \$$. By now photovoltaic powered houses with 1-20 MW have become common place. The advantages claimed are: modular construction, short construction time, small impact on the environment (the surface area required for a given capacity is similar to that of the lake feeding a hydroplant). The Californian Energy Commission is planning a $500 \mathrm{MW}$ plant in five years time.

Europe has been very much less adventurous, but is waking up: The FRG Government is spending $60 \mathrm{M} \$ / \mathrm{a}$, Italy too has a sizable programme while that of the EC is 16 $\mathrm{M} \$ / \mathrm{a}$. Production of amorphous silicon is going up (notably in F and FRG) and will soon reach $1 \mathrm{MW} / \mathrm{a}$ capability. Not that crystalline silicon has been displaced. For flat monocrystals subjected to sunlight concentrated 200 times, efficiencies of $22.6 \%$ have been achieved in the laboratory, $15.2 \%$ in thin films while industry has got up to $12.5 \%$ for bulk production. The comparable figure for amorphous silicon is $6 \%$. Research is concerned with optimising the whole system to give minimum cost. A recent encouraging development is the use of tandem layers, one tailored for the red and the other for the blue and one can expect new materials in the future. Extracting another $1 \%$ efficiency from a system may not at first sight appear to be exciting physics, but to the applied physicist, adding another significant figure to a pure physics number can look far less appealing.

Much of the meeting with the Associate members like that of ACAPPI was concerned with the programme of Industrial Workshop that have now taken a firm hold. New topics were discussed and thought was also given to the potential markets that exist inside university institutions and, the other way round, the services that these institutions might be able to sell to industry - spare beam time on an accelerator for example or high precision analysis. There is a lot that can be done if one has a mind to and preparations are made well in advance. 